\title{
Current Diagnosis and Treatment of Pediatric Latent Tuberculosis Infection
}

\author{
L. A. Hatzenbuehler • J. R. Starke
}

Published online: 28 June 2014

Springer Science + Business Media New York 2014

\begin{abstract}
The goal of diagnosing and treating latent tuberculosis infection (LTBI) in children is to prevent future cases of tuberculosis (TB) disease. In low-prevalence countries, LTBI screening, testing, and treatment are risk based. Testing is limited by lack of a reference standard; both available methods - the tuberculin skin test (TST) and interferon gamma release assays (IGRAs) have significant limitations. The antigens used in IGRAs are not found in BCG-Mycobacterium bovis or most nontuberculous mycobacteria, making these tests more specific for Mycobacterium tuberculosis infection than the TST. The two methods have similar sensitivity, and neither performs well in immunosuppressed children. Children with LTBI are given treatment because it decreases their risk of developing TB disease, and the rate of significant adverse events is low. The most commonly used treatment regimen of 6-9 months of isoniazid is limited in effectiveness by poor adherence. New treatment regimens, using 4 months of rifampin, 3 months of isoniazid and rifampin, or 12 weekly doses of isoniazid and rifapentine, are safe and have significantly higher completion rates.
\end{abstract}

Keywords Latent tuberculosis infection - Tuberculin skin test · Interferon gamma release assays · Isoniazid .

Rifampin · Rifapentine

L. A. Hatzenbuehler · J. R. Starke ( $\square)$

Pediatric Infectious Disease, Department of Pediatrics,

Baylor College of Medicine, 1102 Bates St. Suite 1150,

Houston, TX 77030, USA

e-mail: jrstarke@texaschildrens.org

L. A. Hatzenbuehler

e-mail: hatzenbu@bcm.edu

\section{Introduction}

Tuberculosis (TB) remains one of the most common bacterial infections worldwide, as over $1 / 3$ of the world's population is infected with Mycobacterium tuberculosis [1•]. While the overall incidence of TB has decreased significantly in the United States (U.S.) over the past few decades, it disproportionately affects certain high-risk groups, including ethnic minority and immigrant populations [2]. In the U.S., from 2008 to 2010, 2,660 children and adolescents $<18$ years of age were diagnosed with TB disease, $31 \%$ of whom were foreign-born and $75 \%$ had an international connection through family or residence history [3]. Prior to 2009 , children emigrating to the U.S. from foreign countries often received no testing for TB. Some of these children developed disease or were evaluated and treated for TB after immigration, but many have untreated TB infection and are at risk of developing disease later in life. In addition, many children who were infected with M. tuberculosis within the U.S. or abroad have gone undetected. Because of budget cuts, many local health departments are unable to provide TB screening and testing services for high-risk children in the U.S., which is now the responsibility of primary care physicians. Unfortunately, those children at the highest risk for LTBI in the U.S. are often uninsured and lack a primary care physician or medical home, leaving them unscreened, untested, and untreated for TB [2].

Latent tuberculosis infection (LTBI) means infection with $M$. tuberculosis as indicated by a positive tuberculin skin test (TST) or interferon- $\gamma$ release assay (IGRA) test result, a normal physical exam, and a normal chest radiograph. Most patients with untreated LTBI will never develop tuberculosis (TB) disease. The risk of progression 
from LTBI to disease is highest within the first 6-18 months following initial infection. Otherwise healthy adults have a 5-10\% lifetime risk of progression, while children less than 2 years of age have a $40-50 \%$ risk of progression within 1 year of infection; adolescents have a 10-20\% risk [4••].

The goal of testing for LBTI is to identify and treat children with epidemiologic factors that put them at risk of being infected with $M$. tuberculosis and host factors that put them at risk of developing TB disease [5]. In the pediatric population, this includes children who have had recent contact with an infectious person, have a family history of TB disease or infection, have lived or travelled to a high TB-burden country, or have an immunosuppressive

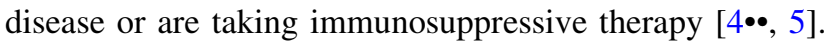

\section{Recent Overview}

Recent studies of childhood LTBI have focused on overcoming the difficulties in its diagnosis and treatment. Because of the limitations of the available testing methods, only children with an identified risk factor for TB infection or rapid progression to $\mathrm{TB}$ disease should be tested. Determining the absolute sensitivity and specificity of the two available methods of testing for LTBI is difficult because there is no reference standard. Their sensitivity has been estimated in studies of patients with culture-positive disease (absolute proof of infection), while their specificity is estimated by their ability to rule out TB disease in lowrisk populations. Most of the early studies comparing the TST and IGRAs were done in adults, but the number and quality of pediatric studies have increased over the last few years. As expected, the IGRAs are more specific than the TST because the antigens used in IGRAs are not found in BCG-M. bovis or most non-tuberculous mycobacteria (NTM); however, the sensitivity of the IGRAs is no higher and, in some studies, lower than that of the TST.

Once a diagnosis of LTBI has been made, children should be treated to prevent the progression of LTBI to TB disease. Children tolerate LTBI treatment better than adults and the effectiveness of treatment is higher, so the benefits of treatment of pediatric LTBI greatly outweigh the risks. The traditionally used regimens of 6-9 months of isoniazid (INH) are limited in effectiveness by poor adherence to this long-term therapy. Alternative LTBI treatment regimens are now available that offer shorter treatment courses (3-4 months), are well tolerated, and have few associated significant adverse events.

\section{Detection of Latent Tuberculosis Infection (LTBI)}

Both the TST and IGRAs are indirect immunodiagnostic tests that measure $\mathrm{T}$ cell response to antigens in $M$. tuberculosis [4••, 5, 6]. Both methods are considered imperfect in that neither provides a definitive diagnosis of LTBI, neither can distinguish between LTBI and TB disease, and both perform poorly in individuals with immunosuppression, including with advanced TB disease [7, 8]. While both tests are valuable tools to detect LTBI, the interpretation of the results should include a clinical evaluation of the child and the individual's risk factors for TB infection or disease.

\section{Tuberculin Skin Test (TST)}

The TST remains the most common diagnostic test for LTBI. It uses PPD (purified protein derivative) tuberculin solution, which is derived from a crude precipitate of more than 200 antigens. The PPD is injected intradermally on the volar surface of the forearm where the antigens stimulate a delayed-type hypersensitivity reaction. Induration (not erythema) is measured along the transverse diameter 48$72 \mathrm{~h}$ after placement $[4 \bullet \bullet, 5]$. TST results can be difficult to interpret. The placement and interpretation of the induration require 2 visits and should be performed by an experienced individual. The reaction size in an individual host can vary by $15 \%$ between tests and the variability in measuring induration among experienced observers also varies by $15 \%$, although it is much greater in untrained persons [9].

The interpretation of the size of a TST induration is based on the individual's risk factors for TB. In individuals whose likelihood of infection is low, specificity takes precedence and an induration of $\geq 15 \mathrm{~mm}$ is considered positive. In those at lower risk, but with an identified risk factor for LTBI, induration $\geq 10 \mathrm{~mm}$ is considered positive. In individuals at highest risk of having LTBI progress to disease (clinical evidence of disease, recent exposure, or immunocompromise), TST sensitivity is paramount, and a reaction $\geq 5 \mathrm{~mm}$ is considered positive [4••].

TST induration can be caused by infection with M. tuberculosis, exposure to NTM, or previous BCG vaccination $[10,11]$. The patient's history and the size of the induration often help determine the cause of the reaction. Most individuals exposed to NTM have indurations $<10 \mathrm{~mm}$, but larger reactions can occur. The most effective way to minimize false-positive results is to avoid testing individuals without a known risk factor for TB.

The bacillus Camille-Guerin (BCG) vaccine is given to infants in high TB-burden countries to reduce the risk of severe forms of TB disease. Many of the antigens in PPD are also expressed by BCG M. bovis, and some vaccinated individuals who are not infected with $M$. tuberculosis express induration in response to the TST. BCG causes induration ranging from 0 to $19 \mathrm{~mm}$, and the effect wanes with time. When BCG is given to school-age children and 
adults, they can retain reactivity for 5-10 years [11-15]. TST specificity in BCG-unvaccinated children is estimated to be 95-100\%. In BCG-vaccinated children, specificity falls to $49-65 \%$, leading to many false-positive results [16].

False-negative TST results also occur in individuals who are unable to mount an adequate immune response. This group includes children who are immunosuppressed by disease (such as advanced HIV infection, advanced TB, cancer, or malnutrition), who receive immunosuppressive treatments (such as corticosteroids, cancer chemotherapy, and immunomodulating agents, especially tumor necrosis factor (TNF) alpha inhibitors), or who recently received a vaccination with live-virus vaccine (particularly, the measles vaccine). False-negative results also can be caused by technical factors such as inadequate storage of the PPD or misinterpretation of the results $[4 \bullet \bullet]$.

Interferon- $\gamma$ release assays (IGRAs)

The IGRAs are ex vivo blood tests that detect T-cell release of interferon-gamma (INF- $\gamma$ ) in response to stimulation by antigens expressed by the M. tuberculosis complex. There are two commercially available IGRA tests: QuantiFERON-TB-Gold-in-Tube (QFT; Cellestis/Qiagen, Carnigie, Australia), and T-SPOT.TB assay (T-SPOT; Oxford Immunotec, Abingdon, United Kingdom). Both tests use early secreted antigenic target 6 (ESAT-6) and culture filtrate protein-10 (CFP-10). QFT measures a response to one additional antigen (TB7.7) [4••, 7]. The antigens in the IGRAs are not encoded in the genomes of $M$. bovis-BCG strains, or most species of NTM, including Mycobacterium avium complex organisms. As a result, IGRAs should be more specific than the TST, yielding fewer false-positive results.

The QFT is an enzyme-linked immunosorbent assay (ELISA) whole blood test that quantifies the amount of INF- $\gamma$ released by sensitized T-cells in response to three antigens. Assays are considered positive if the amount of INF- $\gamma$ in the test sample, after subtracting the amount in the negative control, exceeds a cut-off of $\geq 0.35$ IU (International Units)/milliliter. If there is no detectable INF- $\gamma$ response to the positive control or if the INF- $\gamma$ level in the negative control is too high, the sample is deemed "indeterminate," neither positive nor negative. T-SPOT is an enzyme-linked immunosorbent spot (ELISPOT)-based test that quantifies the number of mononuclear cells producing INF- $\gamma$ in response to two antigens. The result is reported as the number of INF- $\gamma$-producing T-cells. Assays are considered positive if the number of spots in the test sample, after subtracting the number of spots in the negative control, exceeds a threshold, usually 8 spots. Assays with $\leq 5$ spots are considered negative. Assays with 6-7 spots are considered borderline (equivocal), requiring retesting. If the positive control assay has a poor response to stimulation, or if the negative control response is too high, the result is considered "invalid," neither negative nor positive $[4 \bullet \bullet, 7]$.

IGRA Performance in Adults

The test performance of IGRAs was first studied in adults. As there is no reference standard test for LTBI, specificity was estimated in low-risk individuals in a low-prevalence setting. Several studies and meta-analyses have demonstrated IGRA specificity of $95-100 \%$, and unlike the TST, it is not influenced by prior BCG vaccination $[17,18 \bullet \bullet, 19]$. IGRA sensitivity is best estimated among culture- confirmed cases of TB disease (absolute proof of infection). The sensitivity of IGRAs in adults with culture-confirmed disease is comparable to TSTs at $60-90 \%$ (TST sensitivity is $80 \%$ ) with a slight favoring of T-SPOT over QFT. The sensitivity of IGRAs, similar to the TST, is lower in individuals with immunodeficiency. In a meta-analysis evaluating the use of IGRAs compared to the TST in HIVinfected adults, the T-SPOT was slightly more sensitive than QFT (72 vs. $61 \%$ ), but neither was more sensitive than the TST [20].

\section{IGRA Performance in Children}

Fewer studies have evaluated the sensitivity and specificity of IGRAs in children. Because it is difficult to microbiologically diagnose TB disease in children, gradients of exposure to an infectious adult are often used as a surrogate for likelihood of infection to evaluate IGRA performance.

Four systemic reviews and meta-analyses examined

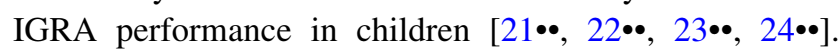
Each analysis was limited by study method heterogeneity and variable clinical definitions of TB. In their meta-analysis, Sun and colleagues included 7 studies that evaluated IGRA specificity in immunocompetent children (0-100\% were BCG vaccinated) [22••]. IGRA specificity was $89 \%$ for BCG-vaccinated children and $95 \%$ for unvaccinated children. TST specificity was $49 \%$ for BCG-vaccinated children and $93 \%$ for unvaccinated children. The percent agreement between the IGRAs and TST was higher in the BCG-unvaccinated children likely due to the influence of false-positive TST results caused by the BCG vaccine. Among 207 children (36\% of whom were BCG vaccinated) with TB risk factors in New York City, only $23 \%$ of children with a positive TST had a positive QFT. QFT better correlated with increased risk of exposure than TST [25]. The influence of gradient of exposure and QFT positivity was also confirmed in a study involving 227 BCGvaccinated children in South Korea [26]. The rate of QFT positivity in unexposed children was $1.5 \%$, compared to a 
rate of $6.9 \%$ in children with casual contact, and $19 \%$ in those with a close contact with TB disease. In the unexposed children (considered at lowest risk of LTBI), QFT specificity was high $(95.5 \%)$. The summary of all the published studies is that the IGRAs are more specific than the TST for children, and the use of IGRAs is favored over the TST in BCG-vaccinated individuals, leading to fewer

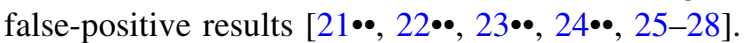

The evaluation of IGRA sensitivity compared to the TST in children is far more challenging, and the best information comes from the pooled data for TB disease diagnosed by culture or clinically, which are included in the pediatric meta-analyses. In children with culture-confirmed disease, Sun and colleagues found a pooled sensitivity of $85 \%$ for QFT, 76\% for T-SPOT, and $85 \%$ for the TST. In individuals who were diagnosed clinically with TB disease, sensitivity was lower for all the three tests (QFT-64 \%, T-SPOT-58 \%, and TST-66\%) [22••]. Although this meta-analysis found no significant difference in test performance between high and low TB-burden settings, two other meta-analyses found the sensitivity of IGRAs to be lower in high burden settings, the

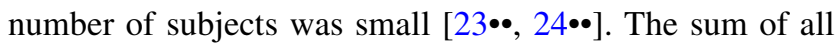
published studies suggests that the IGRAs are not more sensitive than the TST (likely less sensitive in high burden settings) or other measures of determining TB disease in children and cannot be used to rule out TB disease. However, sensitivity for detecting LTBI and TB disease is increasedat the expense of specificity - when both a TST and IGRA are performed (either test being positive), and the use of both tests will increase the rate of positive results in high-risk patients [29].

\section{IGRA Limitations}

Rates of indeterminate/invalid results among children have varied between 0 and $35 \%$, but most studies reported a range of $0-10 \%[7,24 \bullet \bullet, 30 \bullet, 31 \bullet, 32,33]$. The rates seem to be lower with advancements in test technique. Rates of indeterminate/invalid results are increased in children who are immunocompromised (especially with HIV infection), who have poorly controlled inflammatory bowel disease, and who have certain co-infections, including hepatitis, malaria, and helminth infection [34-37]. Indeterminate/invalid IGRA results also occur due to technical error (wrong volume, insufficient mixing, incorrect temperature, prolonged time prior to processing, and laboratory error) [30•, 31•].

There has also been conflicting evidence regarding the performance of IGRAs in infants and children $<5$ years of age, which is thought to be related to an impaired immune response to $M$. tuberculosis in this age group. This is potentially problematic as young children, especially those $<2$ years of age, are at greatest risk of progression to severe forms of TB disease $[16,21 \bullet \bullet, 30 \bullet, 35]$. Critselis and colleagues used the TST and QFT in 761 healthy Greek children in 4 age groups for the diagnosis of LTBI [38•]. Among those $<5$ years age ( 74 were aged $<2$ years old), the authors found a significantly higher number of indeterminate results in younger $(8.1 \%)$ compared to older children $(2.7 \%)$. Debord and colleagues conducted a retrospective analysis of QFT test results in 19 children $<6$ years of age (median 1.52 years; 16 had a TB contact, and most were BCG vaccinated) [39॰]. A trend toward lower QFT sensitivity was observed in the $<2$ years group $(60 \%)$ compared to the $2-$ 5 years group $(100 \%)$, but it did not reach statistical significance $(p=0.087)$. More recent studies have demonstrated better performance of IGRAs in young children. A study in Croatia evaluated 142 healthy BCG-vaccinated children with QFT and the TST following exposure to an infectious adult [30•]. Twenty-four children $(17 \%)$ had a positive TST, and $18(13 \%)$ children had a positive IGRA. Overall test concordance was high $(89 \%, \kappa=0.591)$. In a sub-group analysis, the concordance between QFT and TST among children $<2$ years of age was even higher $(95 \%$, $\kappa=0.828$ ). QFT and TST results were both associated with the degree of exposure. In summary, it appears that the indeterminate/invalid rates are higher in infants and toddlers, but the sensitivity of the IGRAs in these young children has not yet been firmly established.

\section{Current Recommendations for LTBI Testing}

Some of the major differences between the TST and IGRAs are listed in Table 1. The current recommendations for testing children for LTBI are summarized in Table 2 [4••, 40-42•]. Of note

- Only children with a risk factor for LTBI, who are suspected of having TB disease, or are about to undergo significant immunosuppression should be tested with either the TST or an IGRA.

- When evaluating a child for TB disease, or testing those with a TB risk factor who have or are about to undergo significant immunosuppression, both a TST and an IGRA can be done to maximize sensitivity.

- For children $\geq 5$ years of age, either the TST or an IGRA can be used in any situation.

- For children $\geq 5$ years of age who are BCG vaccinated, IGRAs are favored over the TST to maximize specificity. However, if a TST is used and is positive in otherwise low-risk individuals, an IGRA can be done to confirm or refute the TST results.

- In children $<5$ years of age, especially in those $<2$ years of age or with a strong risk factor for TB (such as recent contact to an infectious person), an IGRA should be used cautiously because of concerns about sensitivity. 
Table 1 Comparison of IGRAs and TSTs for the evaluation of LTBI

\begin{tabular}{|c|c|c|}
\hline & TST & IGRA \\
\hline Antigens used & $\sim 200$ & $\begin{array}{l}3 \text { (QFT), } 2 \text { T- } \\
\text { SPOT.TB }\end{array}$ \\
\hline Sample & Intradermal & Blood \\
\hline Patient visits required & 2 & 1 \\
\hline Distinguish LTBI from disease & No & No \\
\hline Cross-reactivity with BCG & Yes & No \\
\hline Cross-reactivity with NTM & Yes & No \\
\hline Differing positive by risk & $\begin{array}{l}\text { Yes }(5-10- \\
15 \mathrm{~mm})\end{array}$ & No \\
\hline Relative cost & Low & Higher \\
\hline $\begin{array}{l}\text { Location of need for trained } \\
\text { personnel }\end{array}$ & "Bedside" & Laboratory \\
\hline $\begin{array}{l}\text { Estimated specificity in BCG- } \\
\text { unvaccinated children }\end{array}$ & $95-100 \%$ & $90-95 \%$ \\
\hline $\begin{array}{l}\text { Estimated specificity in BCG- } \\
\text { vaccinated children }\end{array}$ & $49-65 \%$ & $89-100 \%$ \\
\hline $\begin{array}{l}\text { Estimated sensitivity in BCG-un- } \\
\text { immunized children }\end{array}$ & $75-85 \%$ & $80-85 \%$ \\
\hline $\begin{array}{l}\text { Estimated sensitivity in BCG- } \\
\text { vaccinated children }\end{array}$ & $50-70 \%$ & $60-80 \%$ \\
\hline
\end{tabular}

TST tuberculin skin test, IGRA interferon gamma release assay, QFT QuantiFERON Gold-in-Tube, $B C G$ bacillus Camille-Guerin, NTM non-tuberculous mycobacteria, LTBI latent tuberculosis infection

Table 2 Recommendations for the use of TST and IGRA in children

TST preferred, IGRA acceptable

Children $<5$ years of age

IGRA preferred, TST acceptable

Children $\geq 5$ years of age who have had a BCG vaccine

Children $\geq 5$ years of age who are unlikely to return for the TST reading

Both IGRA and TST

Initial or repeat IGRA is indeterminate/invalid

The initial TST is positive and:

The child is $\geq 5$ years of age and BCG vaccinated

Additional evidence is needed to support an LTBI diagnosis

The initial TST or IGRA is negative and:

There is concern for TB disease

The child is at risk of rapid progression of LTBI to TB disease

TST tuberculin skin test, IGRA interferon gamma release assay, $B C G$ bacillus Camille-Guerin, $L T B I$ latent tuberculosis infection, TB Mycobacterium tuberculosis

\section{Treatment of Pediatric LTBI}

LTBI treatment significantly reduces an individual's lifetime risk of progression to TB disease. Given their higher risk of developing TB disease, children benefit more from LTBI treatment than adults. In addition, children tolerate therapy better and experience fewer adverse events. The efficacy of LTBI treatment regimens is dependent on the susceptibility of the host's M. tuberculosis organism and the individual's completion of the recommended course of treatment $[4 \bullet \bullet, 5]$. The best studied and most often prescribed LTBI regimen for children is 6-9 months of isoniazid (INH). While the efficacy is above $90 \%$, the effectiveness of isoniazid monotherapy is decreased by low completion rates of therapy. At the Tuberculosis Clinic at Texas Children's Hospital, despite counseling families and the child on the importance of LTBI therapy, assisting families in obtaining low-cost INH, and seeing the children at regular clinic follow-up visits, only $49 \%$ of children receiving 9 months of self-administered daily INH therapy complete treatment [43•].

Unfortunately, performing randomized controlled trials of new LTBI regimens for children is difficult and expensive. As a result, most of the information for newer regimens comes from studies performed in adults. It has become the consensus among many childhood TB experts that regimens that are effective for treating TB disease and LTBI in adults are likely to be at least equally effective in children; the essential pediatric data are for pharmacokinetics of drugs, safety, tolerability, adherence to treatment, and completion rates of the regimen. There are several alternative treatment regimens for LTBI that can be considered for children (Table 3).

\section{Isoniazid (INH)}

Isoniazid (INH) is the most extensively studied therapy for the treatment of LTBI in both children and adults. The initial randomized controlled trial of INH therapy was conducted by the United States Public Health Service (USPHS) in the 1950s [44-47]. This trial compared the efficacy of two doses (1.25 vs. $5 \mathrm{mg} / \mathrm{kg})$ and two frequencies (daily or 5 times weekly) of INH given for 12 months. There was a $90 \%$ reduction in TB disease in individuals taking INH $5 \mathrm{mg} / \mathrm{kg}$ either daily or 5 times weekly, a protection that plateaued between 6 and 9 months duration. Further evidence to support INH therapy in children was found by Hsu, who followed a cohort of 1,881 children in Houston with LTBI during and after a 12-month course of daily INH $(10 \mathrm{mg} / \mathrm{kg})$; only 8 children developed TB disease (rate 4.2/1,000) over 30 years, for a protective efficacy of $99 \%$ [48].

\section{Isoniazid, 6 Versus 9 months}

Additional trials have studied the duration of INH therapy. The Scientific Committee on Prophylaxis of the 
Table 3 Comparison of LTBI treatment regimens used in children

\begin{tabular}{|c|c|c|c|c|c|c|}
\hline Medication & Duration & Dose & $\begin{array}{l}\% \\
\text { Adherence }\end{array}$ & Adverse events & Efficacy & Study design \\
\hline INH & $\begin{array}{l}6 \text { or } \\
9 \text { months }\end{array}$ & $\begin{array}{l}\text { Daily: } 10-15 \mathrm{mg} / \mathrm{kg} \\
\text { (max } 300 \mathrm{mg}) \\
2 \times / \text { week: } 20-30 \mathrm{mg} / \\
\mathrm{kg}(\max 900 \mathrm{mg})\end{array}$ & $15-93 \%$ & $\begin{array}{l}\text { Abdominal pain, nausea, vomiting, rash, } \\
\text { AST/ALT elevation, peripheral } \\
\text { neuropathy, } \\
\text { Hepatotoxicity (rare) }\end{array}$ & $\begin{array}{l}9 \text { months of } \\
\text { INH : } 90 \% \\
6 \text { months } \\
60-70 \%\end{array}$ & $\begin{array}{l}\text { Observational } \\
\text { (no children } \\
\text { included in 6- } \\
\text { month } \\
\text { regimen) }\end{array}$ \\
\hline RIF & 4 months & $\begin{array}{l}\text { Daily: } 10-20 \mathrm{mg} / \mathrm{kg} \\
\quad(\max 900 \mathrm{mg})\end{array}$ & $80-94 \%$ & $\begin{array}{l}\text { Staining of secretions, rash, gastrointestinal } \\
\text { upset, neurologic symptoms, bruising, } \\
\text { drug interactions, AST/ALT elevation, } \\
\text { hepatotoxicity (rare), thrombocytopenia } \\
\text { (rare) }\end{array}$ & $\begin{array}{l}\text { Equal to } 6- \\
12 \text { months } \\
\text { of INH }\end{array}$ & Observational \\
\hline $\begin{array}{l}\text { Isoniazid/ } \\
\text { RIF }\end{array}$ & $\begin{array}{l}3 \text { or } \\
4 \text { months }\end{array}$ & $\begin{array}{l}\text { Daily: } 10-15 \mathrm{mg} / \mathrm{kg} \\
\text { (max } 300 \mathrm{mg}) \\
\text { Daily: } 10-15 \mathrm{mg} / \mathrm{kg} \\
\quad(\max 600 \mathrm{mg})\end{array}$ & $78-80 \%$ & As above & $\begin{array}{l}\text { Equal to } 9- \\
12 \text { months } \\
\text { of INH }\end{array}$ & Observational \\
\hline INH/RFP & $\begin{array}{r}12 \text { doses in } \\
16 \text { weeks }\end{array}$ & $\begin{array}{l}\text { Once weekly : } \\
\text { Isoniazid: } \\
15 \mathrm{mg} / \mathrm{kg} \text { rounded } \\
\text { up to the nearest } 50 \\
\text { or } 100 \mathrm{mg} ; 900 \mathrm{mg} \\
\text { maximum } \\
\text { Rifapentine: } \\
\text { 10-14 kg: } 300 \mathrm{mg} \\
14.1-25 \mathrm{~kg}: 450 \mathrm{mg} \\
25.1-32 \mathrm{~kg}: 600 \mathrm{mg} \\
32.1-49.9 \mathrm{~kg}: \\
750 \mathrm{mg} \\
\geq 50 \mathrm{~kg}: 900 \mathrm{mg} \\
\text { maximum }\end{array}$ & $88 \%$ & $\begin{array}{l}\text { INH: as above } \\
\text { RFP: similar to RIF in addition to: } \\
\text { hypersensitivity reaction (rare) }\end{array}$ & $\begin{array}{l}\text { Equal to } \\
9 \text { months } \\
\text { of INH }\end{array}$ & $\mathrm{RCT}$ \\
\hline
\end{tabular}

AST aspartate aminotransferase, $A L T$ alanine aminotransferase, $I N H$ isoniazid, $R I F$ rifampin, $R F P$ rifapentine, $m g$ milligrams, $k g$ kilograms, $R C T$ randomized controlled trial

International Union Against Tuberculosis (IUAT) compared the efficacy of 3-, 6-, and 12-month regimens of INH in 28,000 adult patients with LTBI and fibrotic lesions on chest radiograph [49]. Over 5 years of observation, subjects taking the 12-month regimen had the lowest risk of TB disease $(0.1 \%)$, followed by those taking therapy for 6 months $(0.5 \%)$, and 3 months $(1 \%)$, compared with subjects taking a placebo (1.4\% risk). A review of the data from the UHPHS INH trial showed that 9 months of therapy had a $20 \%$ increased rate of protection compared with 6 months of therapy [46]. However, a Cochrane review of 11 studies involving over 70,000 subjects found no difference in efficacy between 6 and 12 months of INH therapy given daily [50]. The World Health Organization (WHO) currently recommends 6 months of daily INH for treatment of LTBI, while the Centers for Disease Control and Prevention (CDC), the American Thoracic Society (ATS) and American Academy of Pediatrics (AAP) recommend a 9-month course $[4 \bullet \bullet, 5,51]$.
Adherence and Adverse Events with Isoniazid

The effectiveness of INH therapy in children is limited by low completion rates for the entire course of therapy. In a prospective study in South Africa of 236 children $<5$ years of age with LTBI, only $15 \%$ of patients completed therapy and 6 non-adherent individuals developed TB disease [52]. Studies in low TB-burden countries have found INH adherence rates of 51-56\% [43•, 53, 54]. While INH traditionally has been taken daily, a recent large study of children in Houston demonstrated that giving INH twice weekly (20-30 mg/kg) for 9 months under direct observation of health department personnel (DOT) was highly effective, safe, and well tolerated by children of all ages [43•]. Consideration should be given to using DOT, if available, to children with LTBI who are at high risk of developing TB disease, especially recent contacts of cases, infant and toddlers tested for a TB risk factor, and immunocompromised individuals. 
Serious adverse events associated with INH are extremely uncommon in children. An extensive meta-analysis found that of 8,979 children receiving INH $(10-20 \mathrm{mg} / \mathrm{kg} /$ day), 25 (6.8\%) of 369 children having routine laboratory evaluation had asymptomatic mildly increased liver enzymes; one child $(0.003 \%)$ developed jaundice and 68 children $(0.18 \%)$ developed clinical signs of hepatotoxicity; however, treatment had to be discontinued in only 2 children $(0.005 \%)$ [55••]. INH also can cause peripheral neuropathy, which manifests initially as tingling in the distal extremities, caused by increased excretion of pyridoxine (vitamin B6). It is rare in children but more common in adolescents; children with diabetes, malnutrition, or advanced HIV infection; and in breastfeeding infants. These children should be given pyridoxine supplementation when taking INH [6].

\section{Rifampin (RIF)}

Several studies have examined the efficacy, safety, and tolerability of RIF monotherapy for LTBI in adults [5658]. Two small randomized controlled trials comparing the efficacy of 4 months of RIF (10 mg/ $\mathrm{kg}$ up to $600 \mathrm{mg} /$ dose) to 9 months of INH ( $5 \mathrm{mg} / \mathrm{kg}$ up to $300 \mathrm{mg} / \mathrm{dose}$ ) in adults showed comparable efficacy, with improved completion of therapy with RIF (78-81 \%) compared with INH (60-76 \%) [57, 58]. No pediatric randomized controlled trials for RIF therapy have been reported; the data are limited to two pediatric observational studies [59, 60]. RIF therapy was used in a high school in California to treat 157 students with LTBI after their exposure to a classmate with an INH-resistant strain of TB. The students were treated with 6 months of RIF, which was administered 5 days a week at school. After a 2-year follow-up period, no students developed TB disease [59]. More recently, an observational study from Philadelphia compared completion rates between RIF therapy (either 4 or 6 months) and INH therapy (9 months) administered daily at home by the families [60]. Children receiving 4 months of RIF were more likely $(80.5 \%)$ to complete treatment than those taking INH $(53.1 \%)$. This study did not evaluate the efficacy of 6 months versus 4 months of RIF; however, several observational studies in adults suggest a high efficacy associated with 4 months of RIF therapy [61-65].

\section{RIF Adherence and Side Effects}

The studies of adults have shown improved adherence with and better tolerance of RIF regimens compared with INH [61-65]. A large retrospective study involving over 2,000 adults showed that completion rates of RIF therapy given for 4-6 months were three times higher than for 9 months of INH and patients on RIF [65]. In the two observational studies involving children, the RIF regimens were directly observed, leading to excellent adherence rates (88-94\%) $[59,60]$.

Adverse events associated with RIF are infrequent in both adults and children [59-65]. In the large retrospective study involving over 2,000 adult patients, $11.3 \%$ of patients taking 9 months of INH developed any adverse reaction compared to $8.3 \%$ taking $\operatorname{RIF}(p=0.2)$, and $1.8 \%$ of patients taking INH versus $0.08 \%$ of patients taking RIF developed hepatotoxicity $(p<0.001)$ [65]. A large retrospective study from Switzerland included 624 adult patients with LTBI and compared rates of hepatotoxicity (defined as AST or ALT elevation $\geq 3$ times the upper limit of normal with symptoms or $\geq 5$ times the upper limit of normal if asymptomatic) leading to treatment discontinuation [61]. Seven patients out of 426 (1.6\%) taking INH had AST/ALT elevations $\geq 3$ times the upper limit of normal with symptoms compared to 0 patients taking RIF. Elevations of AST/ALT $\geq 5$ times the upper limit of normal occurred in 19 patients taking INH $(4.5 \%)$ compared to 4 patients out of 198 taking RIF (2\%). Hepatotoxicity leading to an interruption of therapy occurred more often in patients taking INH $(6.1 \%)$ compared to RIF (2\%) [61]. In the California observational trial involving 157 adolescents, 4 students (1.3\%) taking RIF had ALT elevations $\geq 2$ times the upper limit of normal which led to a discontinuation of therapy [59]. Minor adverse events occurred in $41(26 \%)$ students including staining of secretions an orange color, mild skin rashes, gastrointestinal complaints, headaches and dizziness, blurred vision, bruising, and nausea and fatigue. Six students had low platelet counts (minimum count of $101 \times 10^{3} / \mathrm{mm}^{3}$ ), and none experienced symptomatic bleeding. Only 18 students who experienced these adverse events temporarily stopped therapy, and only 2 had permanent discontinuation.

The AAP currently recommends 4-6 months of RIF therapy for children with LTBI who have been exposed to an INH-resistant source case (Table 3) [4••]. However, RIF therapy also should be effective for treating a pan-susceptible $M$. tuberculosis infection and there is no inherent reason to limit its use to INH-resistant cases. Prior to the initiation of RIF therapy, a practitioner must consider the following: the high cost of RIF compared with INH (INH is $\$ 4 /$ month at many pharmacies) and the risk of drug-drug interactions. Rifamycins induce the cytochrome CYP-450 system, which increases the metabolism of certain oral contraceptives, anti-epileptic medications, antiretroviral medications, and other medications. Rifamycins should, 
therefore, be used with caution in patients taking any medication with a potential interaction.

\section{Isoniazid and Rifampin}

The combination of INH and RIF given daily for 34 months for treatment of LTBI is used mainly within Europe. Trials performed in both adults and children have evaluated the efficacy and safety of daily INH + RIF regimens compared to INH monotherapy [66-68]. A meta-analysis included 5 randomized controlled trials with 1,900 adult patients with LTBI and compared the efficacy and safety of 9 months of INH to 3 months of INH + RIF [66]. The authors found no difference in efficacy between the two regimens; 39 patients $(4.1 \%)$ who received INH versus 41 patients $(4.2 \%)$ who received INH + RIF developed TB disease. The two regimens also had comparable safety: 46 patients $(4.8 \%)$ who received INH and 48 (4.9 \%) of patients who received INH + RIF developed adverse effects leading to discontinuation of therapy (including hepatotoxicity, rash, and gastrointestinal intolerance). Among 252 patients with LTBI in the British medical system who received 3 months of INH + RIF treatment, and were followed for up to 19 years, only 3 cases of TB disease occurred, a rate of 0.727 cases $/ 1,000$ person years [67].

A randomized study in Greece involved 926 children $<15$ years of age with LTBI who were treated with 9 months of daily INH versus either 3 or 4 months of INH + RIF [68]. Of the children who took 9 months of INH monotherapy, 48 (24\%) developed new radiographic findings (including hilar adenopathy or parenchymal disease) suggestive of possible active disease, compared to 26 $(11.8 \%)$ on 3 months of INH + RIF. These are extremely high rates for both groups and most of the chest radiograph changes were subtle, asymptomatic and resolved without a change in therapy. Patients who took 3 months of INH + RIF had significantly higher rates of adherence to treatment (78-90\%) than those taking INH monotherapy (66\%). Twelve of $200(6 \%)$ children receiving INH monotherapy had mild liver enzyme elevations $(<3$ times the upper limit of normal), compared to 8 of 650 patients $(1.2 \%)$ taking INH + RIF. No children experienced any adverse events during the study leading to a discontinuation of therapy (Table 3 ).

\section{Isoniazid and Rifapentine}

Rifapentine (RFP) is a new rifamycin with a longer halflife (12-15 h) than rifampin allowing for once weekly administration. Recent studies have demonstrated that 12 doses of once weekly INH $(15 \mathrm{mg} / \mathrm{kg}$ up to $900 \mathrm{mg} /$ dose) + RFP (15-25 mg/kg/dose, up to $900 \mathrm{mg} / \mathrm{dose}$ ), administered via DOT, are as effective and safe for treating LTBI as 9 months of daily self-administered INH [69, 70, $71 \bullet \bullet, 72]$. A multi-center, international, randomized controlled trial compared 12 weekly DOT doses of INH + RFP with 9 months of self-administered INH in both adults and children $\geq 2$ years $[71 \bullet \bullet]$. Overall, fewer participants developed TB disease in the INH + RFP group $(7 / 3,986)$ compared with the INH group $(15 / 3,745)$, for a hazard ratio of 0.38 (CI 0.15-0.99). Overall treatment completion rates were higher in individuals taking the INH + RFP regimen $(82.1 \%)$ compared to the INH regimen $(69 \%), p<0.001$. Among those aged 2-14 years, 3 TB cases (0.78 \%) occurred in the INH group compared to 0 cases in the $\mathrm{INH}+\mathrm{RFP}$ group, and completion rates were lower in the INH group ( $81 \%$ completed therapy), compared to the $\mathrm{INH}+\mathrm{RFP}$ group $(88 \%), p<0.003$ [72]

In this trial, individuals in the INH group were more likely than those in the INH + RFP group to develop hepatotoxicity leading to discontinuation of therapy ( $2 \mathrm{vs}$. $0.3 \%,[p<0.01])$. Individuals in the INH + RFP group were slightly more likely to discontinue therapy secondary to hypersensitivity reactions associated with the RFP [71••]. In children ages $2-11$ years of age, no significant differences in the number or severity of adverse reactions were observed between the two treatment regimens (Table 3) [72].

In summary, 12 weekly doses of INH + RFP given via DOT are a safe and well-tolerated regimen for the treatment of LTBI children $>2$ years of age. Because the efficacy data are stronger for older children, it is currently recommended by the AAP and CDC for treatment of LTBI in patients $\geq 12$ years of age and can be used with caution in children ages 2-11 years [73]. Studies are underway to determine if INH + RFP can be given effectively and safely by self-supervision.

\section{LTBI Treatment: Conclusions}

Children are treated for LTBI to decrease their risk of developing TB disease in both the near and distant future. Treatment is highly effective when taken as planned and the rate of significant adverse events is low. The most commonly used treatment regimen of 6-9 months of isoniazid, while highly efficacious in clinical trials, is limited in effectiveness by poor adherence and low completion rates. New treatment regimens, using 46 months of daily rifampin, 3 months of daily isoniazid and rifampin, or 12 once weekly doses of isoniazid and rifapentine under DOT, are safe in children and have significantly higher completion rates. Prior to the 
initiation of LTBI therapy, a practitioner must consider a variety of factors which may affect the likelihood of the child successfully tolerating and completing the chosen regimen. These include medication availability, insurance coverage, medication cost, the time frame available to the family for treatment completion, and the susceptibility of the source case's M. tuberculosis isolate. Given the low frequency of serious adverse events associated with LTBI therapies, routine laboratory monitoring is not recommended for any regimen in an otherwise healthy child [4••, 74]; rather, educating patients and parents about potential adverse events and clinical screening for signs and symptoms of adverse events at regular follow-up visits should be adequate. Children at risk of hepatotoxicity, especially those with existing liver disease or taking other potentially liver toxic medications, should be followed periodically both clinically and with measurement of serum liver enzymes [74, 75].

\section{Conclusion}

Both the TST and IGRAs can be used as tools to diagnose LTBI in children with known risk factors for TB. It is clearly established that the IGRAs are more specific than the TST because they are not influenced by prior BCG vaccination or exposure to most NTM. The TST and IGRAs have comparable sensitivity in older children but there is some concern about their sensitivity in infants and toddlers. As a result, for children less than 4 years of age, TSTs generally are preferred over IGRAs. Sensitivity can be maximized in high-risk children using both methods. However, this will result in decreased specificity and some false-positive results. In children who are immunocompromised by medical conditions or therapy, both the TST and the IGRAs have diminished sensitivity, and the rate of indeterminate/invalid results in the IGRA tests will be increased. Recent studies have identified several treatment regimens for LTBI that have certain advantages over the traditional 6-9 month course of self-supervised INH. These shorter regimens using a rifamycin with or without INH are safe, well tolerated, and result in higher completion rates than seen with the 9-month INH regimen. Efficacy data for these new regimens are not as robust, but current indications are that they are effective, and the increased adherence and completion rates would off-set any small decrease in efficacy.

Disclosure L.A. Hatzenbuehler and Jeffrey R. Starke declare that they have no conflict of interest.

Human and Animal Rights and Informed Consent This article does not contain any studies with human or animal subjects performed by any of the authors.

\section{References}

Papers of particular interest, published recently, have been highlighted as:

- Of importance

-• Of major importance

1. Centers for Disease Control and Prevention. TB in children (global perspective), 2013. Atlanta, GA. Available online at: http://www.cdc.gov/tb/topic/populations/TBinChildren/global. htm Accessed 3 April, 2014. Provides current statistics on the burden of tuberculosis worldwide.

2. Centers for Disease Control and Prevention. Reported tuberculosis in the United States, 2012. Atlanta, GA. http://www.cdc. gov/tb/statistics/reports/2012/pdf/report2012.pdf. Accessed April 3 , 2014. Provides current statistics on the burden of disease of tuberculosis in the United States.

3. Winston CA, Menzies HJ. Pediatric and adolescent tuberculosis in the United States, 2008-2010. Pediatrics. 2012;130(6):e1425-32.

4. •- American Academy of Pediatrics. Tuberculosis. In: Pickering LK, Baker CJ, Kimberlin DW, Long SS, editors. Red Book: 2012 Report of the committee on infectious diseases. Elk Grove Village: American Academy of Pediatrics; 2012. p. 736-759. Provides current recommendations on the diagnosis of pediatric LTBI and TB in the United States.

5. American Thoracic Society. Targeted tuberculin testing and treatment of latent tuberculosis infection. Am J Respir Crit Care Med. 2001;161:S221-47.

6. Pediatric tuberculosis collaborative group. Targeted tuberculin skin testing and treatment of latent tuberculosis infection in children and adolescents. Pediatrics. 2004;114:1175-201.

7. Pai M, Denkinger CM, Kik SV, et al. Gamma interferon release assays for detection of Mycobacterium tuberculosis infection. Clin Microbiol Rev. 2013;27(1):3-20.

8. Bianchi L, Galli L, Moriondo M, et al. Interferon-gamma release assay improves diagnosis of tuberculosis in children. Pediatr Infect Dis J. 2009;28(6):510-4.

9. Menzies D. Interpretation of repeated tuberculin tests. Am J Respir Crit Care Med. 1999;159:15-21.

10. Snider DE Jr. The tuberculin skin test. Am Rev Respir Dis. 1982;125(3 pt 2):108-18.

11. Huebner RE, Schein MF, Bass JB. The tuberculin skin test. Clin Infect Dis. 1993;17(6):968-75.

12. Wang L, Tuner MO, Elwood RK, et al. A meta-analysis of the effect of bacille Calmette Guérin vaccination on tuberculin skin test measurements. Thorax. 2002;27:804-9.

13. Lifshitz M. The value of the tuberculin skin test as a screening test for tuberculosis among BCG-vaccinated children. Pediatrics. 1965;36:624-7.

14. Joncas JH, Robitaille R, Gauthier T. Interpretation of the PPD skin test in BCG-vaccinated children. Can Med Assoc J. 1975;113:127-8.

15. Howard TP, Solomon DA. Reading the tuberculin skin test. Who, when, and how? Arch Intern Med. 1998; 148(11):2457-2457-9.

16. Lewinsohn DA, Lobato MN, Jereb JA. Interferon-gamma release assays: new diagnostic tests for mycobacterium tuberculosis infection, and their use in children. Curr Opin Pediatr. 2010;22 (1):71-6.

17. Deuffic-Burban S, Atsou K, Viget N, et al. Cost-effectiveness of quantiFERON-TB test vs. tuberculin skin test in the diagnosis of latent tuberculosis infection. Int J Tuberc Lung Dis. 2010;14 (4):471-81

18. •• Metcalfe JZ, Everett CK, Steingart KR, et al. Interferongamma release assays for active pulmonary tuberculosis 
diagnosis in adults in low- and middle-income countries: systematic review and meta-analysis. J Infect Dis. 2011; 204(suppl 4):S1120-29. Meta-analysis of 27 observational studies from low and middle income countries, involving both HIV positive and HIV negative adults; found that neither the TST or IGRAs have diagnostic value for the diagnosis of TB in HIV positive adults.

19. Sester M, Sotgiu G, Lange C, et al. Interferon-gamma release assays for the diagnosis of active tuberculosis: a systematic review and meta-analysis. Eur Respir J. 2011;37:100-11.

20. Santin M, Munoz L, Rigau D. Inferferon-gamma release assays for the diagnosis of tuberculosis infection in HIV-infected adults: a systematic review and meta-analysis. PLoS One. 2012;7(3): e32482.

21. • Chiappini E, Accetta G, Bonsignori, et al. Interferon- $\gamma$ release assays for the diagnosis of Mycobacterium tuberculosis infection in children: a systematic review and meta-analysis. Int J Immunopathol Pharmacol. 2012; 25(3): 557-64. Meta-analysis to compare TST and IGRA sensitivity and specificity. The pooled sensitivities were similar between the three methods. IGRA performance showed higher specificity than TST.

22. •• Sun L, Ziao J, Miao Q, et al. Interferon Gamma release assay in diagnosis of pediatric tuberculosis: a meta-analysis. FEMS Immunol Med Microbiol. 2011; 63:165-73. Meta-analysis to compare TST and IGRA sensitivity and specificity. The pooled sensitivities differed between culture confirmed cases and those with LTBI. Agreement between TST and IGRAs in non-BCGvaccinated children was higher than in BCG-vaccinated children. IGRA specificity was higher than TST.

23. • Machingaidze S, Wiysonge CS, Gonzalez-Angulo Y, et al. The utility of an interferon gamma release assay for diagnosis of latent tuberculosis infection and disease in children: A systematic review and meta-analysis. PIDJ. 2011; 30(8):694-700. A review of 20 studies. Found comparable sensitivities between IGRAs and TST, which was reduced in high-incidence settings.

24. • Mandalakas AM, Detjen AK, Hesseling AC, et al. Interferongamma release assays and childhood tuberculosis: systematic review and meta-analysis. Int $\mathbf{J}$ Tuber Lung Dis. 2011; 15 (8):1018-32. Included 33 studies, compared IGRA and TST performance in children with LTBI by gradients of exposure. The sensitivity and specificity of the tests were similar in those with $T B$ disease. The sensitivity was lower in younger children and in those with HIV.

25. Lighter J, Rigaud M, Eduardo R, et al. Latent tuberculosis diagnosis in children by using the QuantiFERON-TB Gold Intube test. Pediatrics. 2009;123:30-7.

26. Chun JK, Kim CK, Kim HS, et al. The role of a whole blood interferon-gamma assay for the detection of latent tuberculosis infection in bacille Calmette-Guerin vaccinated children. Diagn Microbiol Infect Dis. 2008;62:389-94.

27. Ewer K, Deeks J, Alvarez L, et al. Comparison of T-cell based assay with tuberculin skin test for diagnosis of Mycobacterium tuberculosis infection in a school tuberculosis outbreak. Lancet. 2003;361:1168-73.

28. Higuchi H, Harada N, Morit T, et al. Use of QuantiFERON-TB Gold to investigate tuberculosis contacts in a high school. Respirology. 2007;12:88-92.

29. Adetifa IMO, Ota MOC, Jeffries DJ, et al. Commercial interferon gamma release assays compared to tuberculin skin test for diagnosis of latent Mycobacterium tuberculosis infection in childhood contacts in the Gambia. Pediatr Infect Dis J. 2010;29 (5):439-43.

30. • Paviḉ I,Topiç RZ, Raos M, et al. Interferon- $\gamma$ release assay for the diagnosis of latent tuberculosis in children younger than 5 years of age. Pediatr Infect J. 2011; 30(10):866-70. Included 142 BCG vaccinated children $<5$ years of age with LTBI who were exposed to an infectious source case. Showed adequate performance with both TST and IGRA by infectivity of the source case. There was no influence of age on QFT performance.

31. - Cruz AT, Geltemeyer AM, Starke JR, et al. Comparing the tuberculin skin test and T-SPOT.TB blood test in children. Pediatrics. 2011; 127(1):e31-38. Involved a prospective study of 210 patients with variable risk for TB, and compared TST vs. T-SPOT.TB performance by risk category. In high-risk children, concordance was similar, and was not affected by BCG immunization. In intermediate and low-risk children concordance was decreased and impacted by BCG immunization.

32. Connell TG, Ritz N, Paxton, et al. A three-way comparison of tuberculin skin testing, quantiFERON-TB gold and T-SPOT.TB in children. PLoS. 2008; 3(7): e2624.

33. Haustein T, Ridout DA, Hartley JC, et al. The likelihood of indeterminate test result from a whole blood interferon-gamma release assay for the diagnosis of Mycobacterium tuberculosis infection in children correlates with age and immune status. Pediatr Infect Dis J. 2009;28(8):669-73.

34. Tsiaris SJ, Austin J, Toro P, et al. Results of a tuberculosisspecific INF- $\gamma$ assay in children at high-risk for tuberculosis infection. Int J Tuberc Lung Dis. 2006;10(8):939-41.

35. Nicola MP, Davies M-A, Wood K, et al. Comparison of T-SPOT. $T B$ assay and tuberculin skin test for the evaluation of young children at high risk for tuberculosis in a community setting. Pediatrics. 2008;123(1):38-43.

36. Brodsky HO, Ohm J, Zarubova K, et al. Disease activity is an important factor for indeterminate interferon- $\gamma$ release assay results in children with inflammatory bowel disease. J Pediatr Gastroenterol Nutr. 2014;58(3):320-4.

37. Lucas M, Nicol P, McKinnon E, et al. A prospective large-scale study to methods for the detection of latent Mycobacterium tuberculosis infection in refugee children. Thorax. 2010;67:391-8.

38. - Critselis E, Amanatidou, Syridou G, et al. The effect of age on whole blood interferon-gamma release assay response among young children investigated for latent tuberculosis infection. J Pediatr Dis Soc. 2012; 161(4):632-38. Cross-sectional study involving 761 children evaluated for LTBI and were categorized by age groups. INF- $\gamma$ concentrations were higher in younger children, but indeterminate results occurred more frequently.

39. - Debord C, De Lauzanne A, Gourgouillon N, et al. Interferongamma release assay performance for diagnosing tuberculosis disease in 0-to 5-year old children. Pediatr Infect Dis J. 2011; 20 (11):995-97. Evaluated QFT performance in 19 children $<6$ years old. The authors found no indeterminate results in those aged $<2$, and adequate QFT performance.

40. Denkinger CM, Dheda K, Pai M. Guidelines on interferon- $\gamma$ release assays for tuberculosis infection: concordance, discordance or confusion? Clin Microbiol Infect. 2011;17:806-14.

41. Bergamini BM, Losi M, Vaienti F, et al. Performance of commercial blood tests for the diagnosis of latent tuberculosis infection in children and adolescents. Pediatrics. 2009;123(3): e419-24.

42. - Blandinieres A, de Lauzanne A, Guerin-El Khourouj V, et al. QuantiFERON to diagnose Mycobacterium tuberculosis: performance in infants and older children. J Infect. 2013; 67:391-98. Recent prospective study involving 226 immunocompetent children (0-15 years old) compared QFT to TST in the diagnosis of TB disease or LTBI. QFT had $100 \%$ specificity in those without $T B$ disease. QFT sensitivity was influenced by age, and low in children $<5$ years.

43. - Cruz AT, Starke JR. Increasing adherence for latent tuberculosis infection therapy with health-department administered therapy. Pediatr Infect Dis J. 2012; 31(2):193-95. Retrospective analysis of 248 children with LTBI who were started on INH chemoprophylaxis; $75 \%$ of whom completed therapy; the authors highlight barriers to treatment completion and the only variable positively 
associated with adherence was directly observed treatment (DOT).

44. Comstock GW. Isoniazid prophylaxis in Alaskan boarding schools. Am Rev Respir Dis. 1969;100(6):773-9.

45. Comstock GW. Isoniazid prophylaxis among Alaskan Eskimos: a final report of the bethel isoniazid studies. Am Rev Respir Dis. 1979;119:827-30.

46. Comstock GW. How much isoniazid is needed for prophylaxis? Am Rev Respir Dis. 1970;101:780-2.

47. Ferebee SH. Controlled chemoprophylaxis in tuberculosis: a general review. Adv Tuberc Res. 1970;17:28-106.

48. Hsu KH. Thirty years after isoniazid. JAMA. 1984;251(10): $1283-5$.

49. International Union Against Tuberculosis Committee on Prophylaxis. Efficacy of various durations of isoniazid preventive therapy for tuberculosis: five years of follow-up in the IUAT trial. Bull World Health Organ. 1982;60(4):555-64.

50. Smieja M, Marchetti C, Cook D, et al. Isoniazid for preventing tuberculosis in non-HIV infected persons. Cochrane Database Syst Rev. 1999;1:1-28.

51. Centers for Disease Control and Prevention. Targeted Tuberculin Testing and Treatment of Latent Tuberculosis Infection. MMWR: recommendations and reports. 2000; 49(RR-6):1-CE 7. http:// www.cdc.gov/mmwr/pdf/rr/rr4906.pdf. Accessed 3 April 2014.

52. Marias BJ, van Zyl S, Schaaf HS, et al. Adherence to isoniazid preventive chemotherapy: a prospective community based study. Arch Dis Child. 2006;91:762-5.

53. Van Zyl S, Marais BJ, Hesseling AC, et al. Adherence to antituberculosis chemoprophylaxis and treatment in children. Int $\mathbf{J}$ Tuberc Lung Dis. 2006;10(1):13-8.

54. Bibi H, Weiler-Ravell D, Shoseyov D, et al. Compliance of treatment of latent tuberculosis infection in a region of Israel. Isr Med Assoc J. 2002;4:13-6.

55. •• Donald PR. Antituberculous drug-induced hepatotoxicity in children. Pediatr Rep. 2011;3(16):51-64. Thorough review of antituberculous drug associated adverse events and hepatotoxicity in children. All regimens are considered safe with improved tolerance compared to adults.

56. Hong Kong Chest Service/Tuberculosis Research Center. Madras/British Medical Research Council. A double-blind, placebo-controlled trial of three antituberculous chemoprophylaxis regimens in patients with silicosis in Hong Kong. Am Rev Respir Dis. 1992;145:36-41.

57. Menzies D, Dion MJ, Rabniovitch B, et al. Treatment completion and costs of a randomized trial of rifampin for 4 months versus isoniazid for 9 months. Am J Respir Crit Care Med. 2004;170: 445-9.

58. Menzies D, Long R, Trajman A, et al. Adverse events with 4 months rifampin or 9 months isoniazid as therapy for latent TB infection: results of a randomized trial. Ann Intern Med. 2008;149: 689-97.

59. Villarino ME, Ridzon R, Weismuller PC, et al. Rifampin preventive therapy for tuberculosis infection: experience with 157 adolescents. Am J Respir Crit Care Med. 1997;155:1735-8.

60. Daskalaski I, Dogbey MC, Tolbert-Warren C, et al. Tolerability of rifampin monotherapy for latent tuberculosis infection in children. Pediatr Infect Dis J. 2011;30:1014-5.
61. Fresard I, Bridevaux P, Rochat T, et al. Adverse effects and adherence to treatment of rifampicin 4 months vs isoniazid 6 months for latent tuberculosis. Swiss Med Wkly. 2011;141:1-5.

62. Lardizabal A, PassannanteM, Kojakali F, et al. Enhancement of treatment completion for latent tuberculosis infection with 4 months of rifampin. Chest. 2006;130(6):1712-17.

63. Fountain FF, Tolley EA, Jacobs AR, et al. Rifampin hepatotoxicity associated with treatment of latent tuberculosis infection. Am J Med Sci. 2009;337(5):317-20.

64. Menzies D, Long R, Trajman A, et al. Adverse events with 4 months of rifampin therapy or 9 months of isoniazid therapy for latent tuberculosis infection. Ann Int Med. 2008;149(10):689-98.

65. Page KR, Sifakis F, Mondes de Oca R, et al. Improved adherence and less toxicity with rifampin vs isoniazid for treatment of latent tuberculosis. Arch Intern Med. 2006;166:1863-70.

66. Ena J, Valls V. Short-course therapy with rifampin plus isoniazid compared with standard therapy with isoniazid, for latent tuberculosis infection: a meta-analysis. Clin Infect Dis. 2005;40:6706.

67. Bright-Thomas R, Nandwani S, Smith J, et al. Effectiveness of 3 months of rifampicin and isoniazid chemoprophylaxis for the treatment of latent tuberculosis infection in children. Arch Dis Child. 2010;95:600-2.

68. Spyridis NP, Spyridis PG, Gelesme A, et al. The effectiveness of a 9-month regimen of isoniazid alone versus 3- and 4- month regimens of isoniazid plus rifampin for treatment of latent tuberculosis infection in children: results from an 11-year randomized trial. Clin Infect Dis. 2007;45:715-22.

69. Martinson NA, Barnes GL, Moulton LH, et al. New regimens to prevent tuberculosis in adults with HIV infection. N Engl J Med. 2011;365:11-20.

70. Schechter M, Zajdenverg R, Falco G, et al. Weekly rifapentine/ isoniazid or daily rifampin/pyrazinamide for latent tuberculosis in household contacts. Am J Respir Crit Care Med. 2006;173:922-6.

71. •• Sterling TR, Villarino ME, Borisov AS, et al. Three months of rifapentine and isoniazid for latent tuberculosis infection. N Engl J Med. 2011;365(23):2155-66. Randomized controlled trial comparing 3 months of directly observed once weekly therapy with isoniazid (INH) and rifapentine (RFP) versus 9 months of self-administered isoniazid. The study showed equal efficacy between groups, with improved adherence and fewer adverse events experienced by the INH/RFP group.

72. Villarino ME, Scott NA, Weis SE, et al. New treatment for preventing tuberculosis in children: a 3 month, 12-dose regimen of rifapentine and isoniazid [Poster presentation]. Infectious Disease (ID) Week, Session 182: Clinical management of infectious diseases. 2012; Presentation \#1323.

73. Centers for Disease Control and Prevention. Recommendations for the use of isoniazid-rifapentine regimen with direct observation to treat latent Mycobacterium tuberculosis infection. MMWR. 2011;26(48):1650-3.

74. O'Brien RJ, Long MW, Cross FS, et al. Hepatotoxicity from isoniazid and rifampin among children treated for tuberculosis. Pediatrics. 1983;72(4):491-9.

75. Cruz AT, Ahmed A, Mandalakas AM, et al. Treatment of latent tuberculosis infection in children. J Pediatr Infect Dis Soc. 2013;2 (3): $248-58$. 